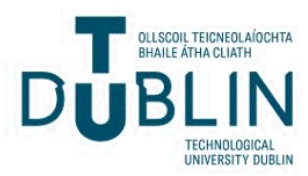

Technological University Dublin ARROW@TU Dublin

\section{Integration of antennas with sun-tracking solar panels}

\author{
Adam Narbudowicz \\ Technological University Dublin, adam.narbudowicz@mydit.ie
}

Follow this and additional works at: https://arrow.tudublin.ie/ahfrcart

Part of the Engineering Commons

\section{Recommended Citation}

Narbudowicz, A., O'Conchubhair, O. \& Ammann, M. (2016). Integration of antennas with sun-tracking solar panels. IEEE Xplore Digital Library.2016. doi:10.1049/el.2016.1040.

This Article is brought to you for free and open access by the Antenna \& High Frequency Research Centre at ARROW@TU Dublin. It has been accepted for inclusion in Articles by an authorized administrator of ARROW@TU Dublin. For more information, please contact arrow.admin@tudublin.ie, aisling.coyne@tudublin.ie, gerard.connolly@tudublin.ie.

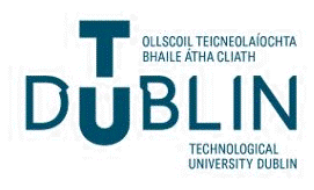




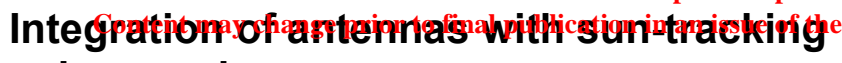 solar panels}

\author{
A. Narbudowicz, O. O'Conchubhair, M. J. Ammann and \\ D. Heberling
}

This letter proposes a low-cost solution for antennas integrated into solar cells, allowing their implementation in solar tracking installations. The proposed passive device autonomously adjusts the phase shift of a twoelement array proportional to the panel rotation. Consequently, the main beam is steered to compensate for panel rotation and maintain a radio link. The proposed device operates for a rotation of $\pm 45^{\circ}$ with an amplitude variation of $\leq 0.4 \mathrm{~dB}$ at $2.51 \mathrm{GHz}$.

Introduction: Significant reductions in the operating costs of remotely located wireless devices have resulted in increased use of photovoltaics in radio systems [1]. The integration of antennas with photovoltaics is thus attractive, with many designs reported in the literature, e.g. [2 - 4]. These solutions are however designed only for fixed solar panels, i.e. ones where the solar illumination angle cannot be optimized. Implementing solar tracking ensures that the solar incidence angle is within the cell acceptance angle for most of the day thus maximising solar energy generation. However this solar cell movement would degrade the telecommunication link, as the main beam would also track the sun. A classical beamsteering solution could be implemented, however the electronically-controlled phase shifters and control unit would significantly increase the installation cost.

This letter proposes a passive phase shifter, which allows autonomous phase offset adjustment to compensate for the cell movement. This is achieved by adjusting the signal path lengths during solar tracking, when a motor (or other device) rotates the panel. The solution was successfully tested with two printed Inverted-F antennas integrated with a solar cell as described in [4]. The device allows adjustment in either elevation or azimuth; a $2 \times 2$ array with a set of cascaded phase shifters allows $2 \mathrm{D}$ steering. The device can be used to stabilize the beam for any rotating or rolling platform, such as airborne nodes or maritime platforms (e.g. buoy or small vessel). The device is intended as a low-cost solution, hence the use of standard FR-4 materials and off-the-shelf solar cells.

Design: The phase shifter is built using two PCBs, comprising a fixed part (A) and a rotating part (B) as shown in Fig. 1a. When pressed together, the two boards form a stripline, i.e. the fixed part (A) forming the lower half of the stripline and the rotating part (B) forms the upper half. The manufacturing allows rotation between the two parts along an axis (shown as a black dot-dashed line), with the angle of rotation denoted as $\gamma$. The fixed part (A) is firmly mounted to the installation base, while the rotating part (B) is mounted directly on the movable section of the solar cell. While the solar cell rotates to track the sun, the phase shifter autonomously adjusts the phase shift $\Delta_{\mathrm{ph}}$, thus rotating the beam direction to compensate for the cell movement and maintaining the communication link.

Each PCB is $1.5 \mathrm{~mm}$ thick, with $\varepsilon_{\mathrm{r}}=4.3$ and $\tan \delta=0.025$. The circular parts, seen in Fig. 1a, are of radius $\mathrm{R}_{1}=20 \mathrm{~mm}$. The rectangular extension in part (A) is protruding by $22 \mathrm{~mm}$ and is $20 \mathrm{~mm}$ wide. The two extensions in part (B) are protruding by $10 \mathrm{~mm}$ and are also $20 \mathrm{~mm}$ wide. When the shifter is in the $\gamma=0^{\circ}$ position its footprint can be enclosed within a $68 \times 44 \mathrm{~mm}$ rectangle.

Port 1 of the shifter (SMA connector) is located on the fixed part and is connected to a $50 \Omega$ stripline ( $1.4 \mathrm{~mm}$ wide). A simple T-junction is used to split the signal, with a $\lambda / 4$ transformer to ensure good matching. The two output branches are shaped as a $140^{\circ}$ arc of radius $R_{2}=12 \mathrm{~mm}$. On the rotating part (B) a corresponding arc is milled, which is $300^{\circ}$ and also of radius $R_{2}$. With the two parts pressed together, an electrical contact between the two arcs is formed, preserving the continuity of the stripline. A change of angle $\gamma$ will lengthen one branch and shorten the other, effectively yielding a phase shift $\Delta_{\mathrm{ph}}$ that is:

$$
\Delta_{p h}=\frac{2 \pi R_{2}}{\lambda_{e f f}} 2 \gamma
$$

To cite the paper please use the doi provided on the Digith
where $\lambda_{\text {eff }}$ is the effective wavelength in the stripline.

This phase shift is used to drive two printed inverted-F antennas (seen in Fig. 1b), integrated with the solar cell as described in [4]. The antennas have a centre frequency at $2.513 \mathrm{GHz}$ ( $\mathrm{S}_{11}$ not shown for brevity) and are separated by $77 \mathrm{~mm}$, which is determined by the cell's bus bar spacing. Each antenna is milled on a $0.4 \mathrm{~mm}$ thick FR-4 substrate with dimensions of: $H=6.4 \mathrm{~mm}, L=25.3 \mathrm{~mm}, S=3 \mathrm{~mm}$ and $W=1 \mathrm{~mm}$. The solar cell doubles as antenna's ground plane and has dimensions of $156 \times 156 \mathrm{~mm}$.

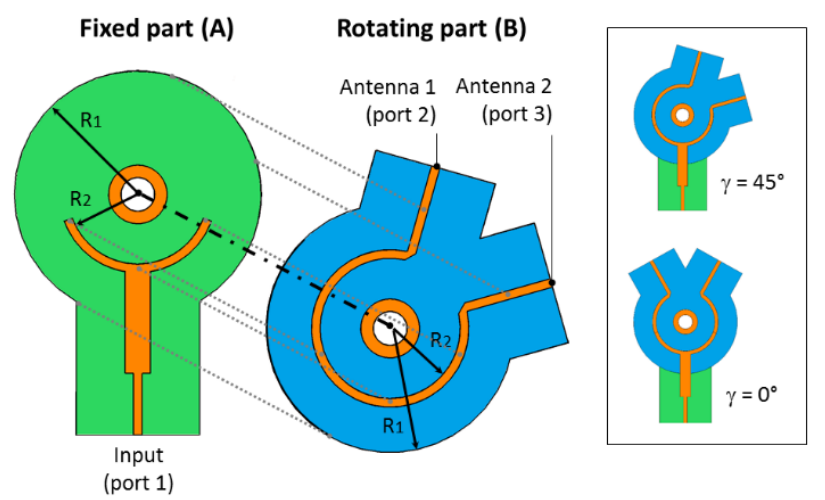

(a)

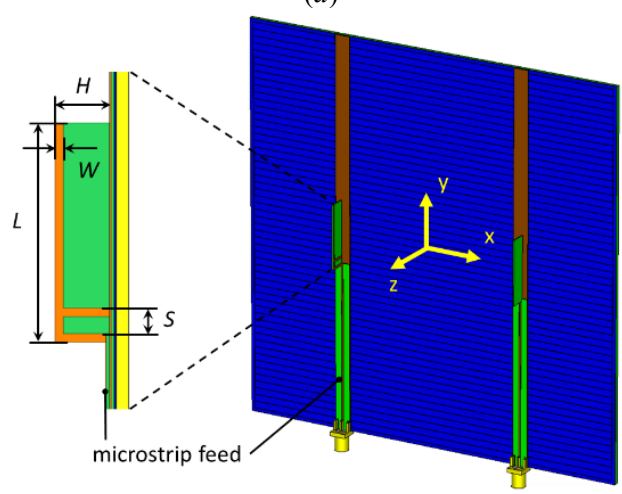

(b)

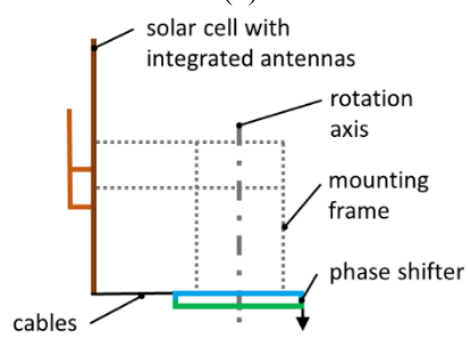

(c)

Fig. 1 (a), The proposed phase shifter for passive phase correction (b) prototyped antennas integrated with solar cell and (c) schematic of the complete structure.

Results: The phase shifter and antennas were prototyped and measured. Fig. 2 demonstrates the measured reflection and transmission coefficients of the phase shifter for different values of $\gamma$. The $-10 \mathrm{~dB}$ bandwidth for the worst case of $\gamma=45^{\circ}$ spans from $2.49 \mathrm{GHz}$ to $2.87 \mathrm{GHz}(14 \%)$, i.e. much broader than the bandwidth of the antenna used for testing [4]. The transmission at $2.512 \mathrm{GHz}$ varies between $-4.7 /-4.9 \mathrm{~dB}$ for $\gamma=0^{\circ}$ to $5.2 /-5.5 \mathrm{~dB}$ for $\gamma=45^{\circ}$. The relatively high losses are due to the use of low-cost FR-4 material and can be improved if more RF-suitable material is used. More importantly, the rotation from $\gamma=0^{\circ}$ to $\gamma=45^{\circ}$ introduced only $0.5 \mathrm{~dB}$ of additional losses, which proves the usefulness of the proposed system. Fig. 3 demonstrates both measured and simulated phase and amplitude variations between $\mathrm{S}_{21}$ and $\mathrm{S}_{31}$ as a function of $\gamma$. It can be seen that the performance has a linear phase with good agreement between simulation and measurement. The amplitude variation between the two antenna ports is for all investigated cases $\leq 0.4 \mathrm{~dB}$. This is considered a good result for a proof-of-concept prototype. The variation 
This article has been accepted for publication in a future issue of this journal, but has not been fully edited.

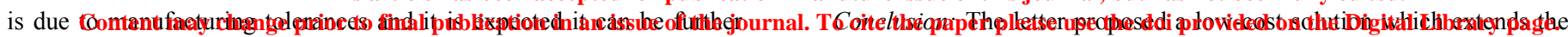
decreased with more advanced manufacturing procedures.

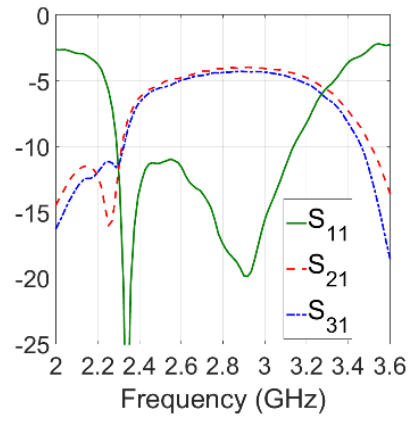

(a)

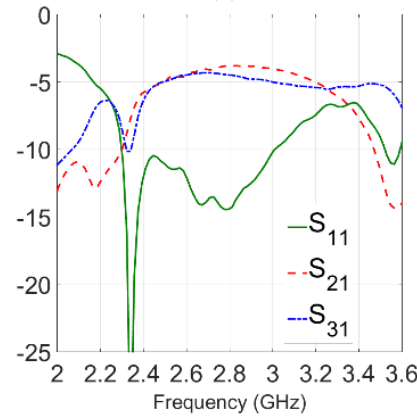

(c)

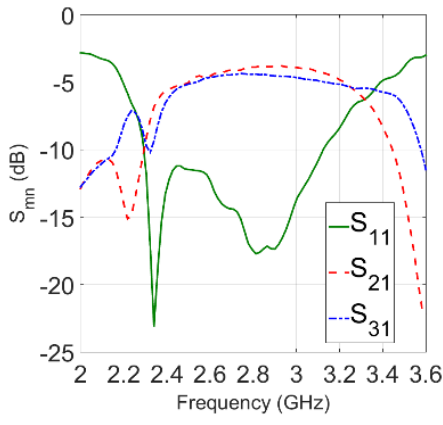

(b)

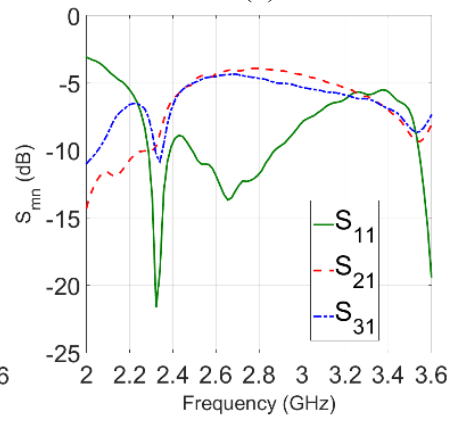

(d)

Fig. 2 S-parameters of the phase shifter for various rotations of $\gamma:$ a) $\gamma=0^{\circ}$; b) $\gamma=15^{\circ}$; c) $\gamma=30^{\circ}$; d) $\gamma=45^{\circ}$.

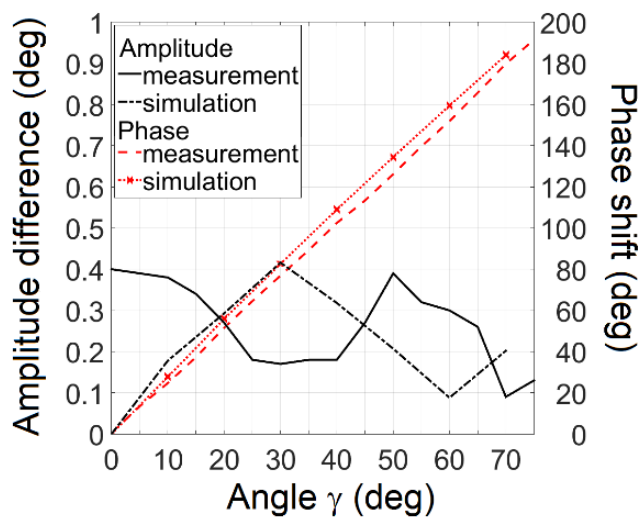

Fig. 3 Phase and amplitude differences between $S_{21}$ and $S_{31}$ of the phase shifter at $2.51 \mathrm{GHz}$.

The array realized gain patterns with the proposed shifter were measured up to $\gamma= \pm 45^{\circ}$, which allows steering over a $90^{\circ}$ span. It should be noted, that for greater angles the dependency between beam direction and phase shift becomes less linear, due to both array factor and radiation pattern of each element. Fig. 4 demonstrates the realized gain patterns for the whole system, i.e. incorporating all the mismatch and ohmic losses. The coordinate system is aligned with antennas. It can be seen, that the beam is tilted as expected, with the tilt angle precisely compensating for the rotation within $\pm 45^{\circ}$. Since the device is intended to compensate for the movement, the beam tilt is in the opposite direction to the rotation $\gamma$. The measured gain varies between $3 \mathrm{dBi}\left(\gamma= \pm 45^{\circ}\right)$ and $2 \mathrm{dBi}\left(\gamma=0^{\circ}\right)$. This is due to the very wide beam of PIFA antennas and losses in the FR4 material. With increased rotation angle $\gamma$, the sidelobes also increase reaching $0.3 \mathrm{dBi}$ at $-65^{\circ}$ for $\gamma=-45^{\circ}$ and $0.1 \mathrm{dBi}$ at $+55^{\circ}$ for $\gamma=+45^{\circ}$. This is due to the antenna spacing being slightly greater than $\lambda_{0} / 2$. This distance was imposed by the distance between the DC collecting bus bars in an off-the-shelf cell. For a custom-designed solar cell this performance can be easily optimized. operability of antennas integrated into solar cells, allowing their implementation in solar tracking systems. The phase shifter autonomously adjusts its phase in harmony with the solar cell tilt angle, thus tilting the beam within a range of $\pm 45^{\circ}$ and compensating for solar cell orientation. The device allows adjustment in either elevation or azimuth, with a possible extension to $2 \mathrm{D}$ steering with $2 \times 2$ array and a set of cascaded phase shifters. Overall, the device allows more effective power collection without compromising the radio link.

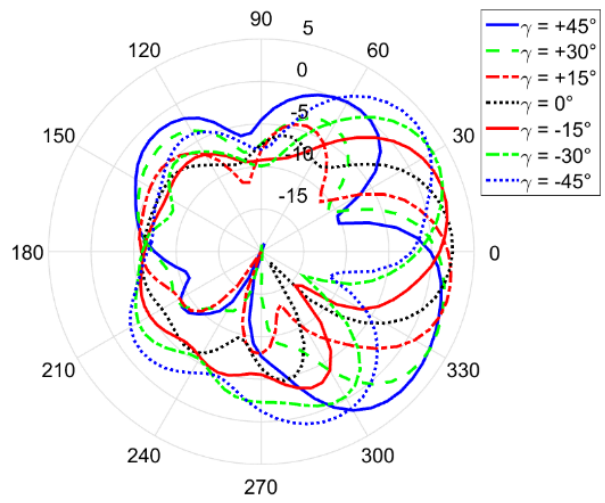

Fig. 4 Absolute realized gains for the proposed antenna at $2.51 \mathrm{GHz}$ in the xz-plane. The results incorporate all losses in the $R F$ chain, including the impact of the proposed phase shifter.

Acknowledgments: This work was supported by Irish Research Council under "ELEVATE" Fellowship scheme (grant ELEVATEPD/2014/79) and in part by Science Foundation Ireland (grant 13/RC/2077).

A. Narbudowicz, O. O'Conchubhair, and M. J. Ammann (Antenna and High Frequency Research Centre, School of Electrical \& Electronic Engineering, Dublin Institute of Technology, Kevin St., Dublin 8, Ireland)

E-mail: adam.narbudowicz@dit.ie

D. Heberling (Institute of High Frequency Technology, RWTH Aachen University, 52056 Aachen, Germany)

A. Narbudowicz: also with Institute of High Frequency Technology, RWTH Aachen University, 52056 Aachen, Germany

\section{References}

1 Olatomiwa, L. J., Mekhilef, S, Huda A. S. N.: 'Optimal sizing of hybrid energy system for a remote telecom tower: A case study in Nigeria', In Proc.: Energy Conversion (CENCON), 2014 Conference on, pp. 243247, 13-14 Oct. 2014, Johor Bahru, Malaysia

2 Dreyer, P., Morales-Masis, M., Nicolay, S., Ballif, C., PerruisseauCarrier, J.: 'Copper and Transparent-Conductor Reflectarray Elements on Thin-Film Solar Cell Panels', IEEE Trans. on Ant. and Prop., 2014, 62, (7), pp. 3813-3818, doi: 10.1109/TAP.2014.2316539

3 Caso, R., D'Alessandro, A., Michel, A., Nepa, P.: 'Integration of Slot Antennas in Commercial Photovoltaic Panels for Stand-Alone Communication Systems', IEEE Trans. on Ant. and Prop., 2013, 61, (1), pp. 62-69, doi: 10.1109/TAP.2012.2220111

4 O'Conchubhair, O., McEvoy, P., Ammann, M. J.: 'Integration of Antenna Array with Multicrystalline Silicon Solar Cell', IEEE Ant. and Wireless Prop. Letters, 2015, 14, pp. 1231-1234, doi: 10.1109/LAWP.2015.2399652 